\title{
Correlación entre indicadores bibliométricos en revistas de Web of Science y Scopus
}

\author{
José Antonio SALVADOR-OLIVÁN \\ jaso@unizar.es \\ Carmen AGUSTín-LACRUZ \\ cagustin@unizar.es \\ Universidad de Zaragoza \\ Departamento de Ciencias de la Documentación e Historia de la Ciencia
}

Recibido: Septiembre 2015

Aceptado: Noviembre 2015

\begin{abstract}
Resumen: Este artículo analiza el grado de correlación existente entre diferentes indicadores bibliométricos obtenidos de las revistas indizadas en 2013 enWoS y Scopus. Se han seleccionado los indicadores Factor de Impacto (FI), Factor de Impacto 5 años (FI5), Inmediatez, Eigenfactor Score (ES), Influencia del Artículo (AIS), ScimagoJournal Rank(SJR), citas/documentos en 2 años (FIScopus), índice H, Impacto por Publicación (IPP) e Impacto Normalizado por Artículo (SNIP). El número total de revistas coincidentes en ambas bases de datos fue de 10.700, de las que se seleccionaron para el análisis dela correlación 9.657 que contenían todos los indicadores. Se aplicó el coeficiente de correlación de Spearman mostrando en algunos indicadores valores muy altos, tanto de manera global como en las áreas de ciencias y ciencias sociales, evidenciando validez convergente, lo que plantea si son necesarios distintos indicadores cuando realmente miden lo mismo. Solo el índice $\mathrm{H}$ presentólos valores más bajos,ofreciendo información complementaria al resto de indicadores.
\end{abstract}

Palabras clave: Correlación; Indicadores bibliométricos; Indicadores de popularidad; Indicadores de prestigio; Revistas científicas; Scopus; Web of Science.

\section{Correlation between bibliometric indicators in Web of Science y Scopus journals}

\begin{abstract}
This article compares the correlation between bibliometric indicators obtained from journals indexed in the Web of Science (WoS) and Scopusdatabases in 2013. Indicators selected for analysis include impact factor (FI), 5-year impact factor (5yrFI), Inmediacy index, Eigenfactor Score (EF), Article Influence Score (AIS), SCImago Journal Rank (SJR), cites/docs 2 years (FIScopus), h-index, Impact Per publication (IPP) and Source Normalized Impact per Paper (SNIP).From 10700 journals listed in both databases, 9657 for analysis were selected because they contained all indicators.Correlations were determined for each pair of indicators and were tested with Spearman's rho. The results shown that the correlations between several indicators are high and that they exhibit similar values in science and social sciences, providing evidence of convergent validity. Only $\mathrm{H}$-index presentedthe lowest valuesproviding complementaryinformation to other indicators.
\end{abstract}


Keywords: Bibliometric indicators; Correlations; Popularity indicators; Prestige indicators; Scientific journals; Scopus; Web of Science.

\section{INTRODUCCIÓN}

La última etapa de una investigación consiste en la difusión y publicación de los resultados, habitualmente, en una revista científica. ¿En qué revista publicar los resultados de una investigación?, ¿Cuál es la que tiene más prestigio o está entre las más influyentes de una determinada disciplina científica?. Estas son las cuestiones que se plantean habitualmentelos investigadorescuando decidenpublicar los resultados de su estudio o incluso leer los artículos publicados en las revistas de mayor impacto y que contienen los descubrimientos más importantes. También los responsables de la gestión de las bibliotecas necesitan esta información para decidir mantener o cancelar la suscripción a una o más revistas.

Dos son los métodos utilizados para la evaluación de la calidad de las revistas, la reputación basada en la opinión de expertos y el recuento de citas que reciben (Sellers; Mathiesen; Perry; Smith, 2004). El principal inconveniente de la evaluación por expertos señalado en la literatura ha sido su carácter subjetivo, de ahí que se haya utilizado con mayor frecuenciael segundo, necesario para comprender el impacto científico de las revistas (Rizkallah; Sin, 2010).

El impacto científico es un constructo multidimensional que no se puede medir con un único indicador (Bollen, 2009), de ahí que se hayan desarrollado varios indicadores bibliométricos para medir la categoría científica de una revista. Esta viene determinada por dos factores, la popularidad medida a través del número de citas que recibe una publicación, y el prestigio, que pondera las citas según la procedencia de las revistas citantes (Franceschet, 2010).

El primer indicador de popularidad creado y ampliamente aceptado por la comunidad científica para evaluar la calidad de revistas fue el Factor de Impacto (FI). Propuesto por Eugene Garfield (Garfield, 1955) para medir la importancia relativa de las revistas dentro de un campo científico basada en las citas que recibía (Garfield, 1972; Garfield, 2006), se construyó a partir de las bases bibliográficas creadas por el Institute for Scientific Information (ISI).

El FI de una revista para un año determinado es el número medio de citas que reciben en ese año los artículos publicados en dicha revista durante los dos años anteriores. En la actualidad se calcula para las revistas indizadas en la base de datos Web of Science (WoS) de Thomson Reuters y se utiliza como medida para evaluar la importancia de una revista a lo largo de los años, siendo actualizado todos los años y fácilmente accesible (Fersht 2009) en la publicación Journal Citation Reports (JCR).

Es una evidencia en la literatura que el FI difiere entre diversas disciplinas científicas debido principalmente a que tienen hábitos y prácticas de citación 
distintas, a un lapso de tiempo diferente entre la publicación y la citación posterior y a diferencias en la fracción de citas a la literatura indizada en el JCR, siendo esta última causa la que más contribuye a la diferencia entre los campos de conocimiento (Althouse; West; Bergstrom, 2009).

A pesar de sus limitaciones y de numerosas críticas recibidas (Dong; Loh; Mondry, 2005; Bordons; Fernández; Gómez, 2002; Harter; Nisonger, 1997; Durieur; Gevenois, 2010), el FI se sigue utilizando para ordenar y clasificar las revistas por su impacto en la comunidad científica, si bien se han desarrollado otros indicadores alternativos con el ánimo de limar sus inconvenientes al tener en cuenta otros aspectos que ofrecen información complementaria sobre la calidad e impacto de las revistas.

El JCR proporciona otros dos indicadores bibliométricos:

- Factor de Impacto 5 años: Utiliza la misma fórmula que el FI pero ampliando la ventana de citación a 5 años, intentando de esta manera no perjudicar aquellas áreas en las que el tiempo que transcurre entre la publicación y la cita es mayor y no se produce un envejecimiento tan rápido de la literatura (Jacsó, 2009).

- Índice de Inmediatez: Mide la prontitud o rapidez con que se citan los artículos de una revista determinada. Se calcula dividiendo el número de citas recibidas entre los artículos publicados en un determinado año por el número de artículos publicados en esa revista en el mismo año. Está influenciado, entre otros factores, por los retrasos y la frecuencia de la publicación. Permite identificar revistas punteras en áreas de investigación emergentes y que contienen trabajos de amplia repercusión.

Web of Science proporciona otros dos indicadores bibliométricos, calculados por eigenfactor.org:

- Eigenfactor (EF): Mide la influencia total de una revista en la literatura académica y en su cálculo intervienen factores que ignora el FI: el prestigio de las revistas citantes y las diferencias en los patrones de citación entre disciplinas (Bergstrom, 2007). Se calcula para un periodo de 5 años, excluye las autocitas y tiene en cuenta las citas recibidas en revistas y otras publicaciones tanto en Ciencias Sociales como en Ciencia dando más peso a aquellas citas que proceden de artículos que a su vez son más citados. Tal y como se indica en su web, "TheEigenfactor ${ }^{\circledR}$ score of a journalisanestimate of thepercentage of time thatlibraryusersspendwiththatjournal" (University of Washington, 2012), por lo que el tiempo que un investigador emplea en cada revista es una medida de la importancia de dicha revista dentro de la red de citas académicas (Bergstrom y otros, 2008). Este índice no se normaliza por el número de artículos publicados, por lo que las revistas que más 
publicanalcanzan valores más altos. La suma de valores de todas las revistas es igual a 100.

- Influencia del Artículo (ArticleInfluence Score, AIS): Se calcula dividiendo el Eigenfactor por el número de artículos publicados en la revista. Al ajustar el número de citas recibidas por el número de artículos publicados en la revista, mide la influencia media de los artículos de una revista, siendo comparable conceptualmente al Factor de Impacto de 5 años (Bergstrom; West; Wiseman, 2008). La media de este indicador es igual a 1.

En 2004, Reed Elsevier lanzó al mercado la base de datos Scopus, de cobertura multidisciplinar, que se empezó a utilizar también para obtener otros indicadores bibliométricos como:

- ScimagoJournal Rank (SJR): Creado en 2007 por el grupo de investigación SCImago, se calcula con las revistas incluidas en la base de datos Scopus y mide la influencia o el prestigio de las revistas. A diferencia con el FI, amplía la ventana de citación a 3 años, otorga más peso a las citas procedentes de revistas con mayor prestigio (aquellas que, a su vez, reciben más citas), limita el número de autocitas y tiene en cuenta en el denominador todos los documentos publicados en la revista (González-Pereira; Guerrero-Bote;MoyaAnegón, 2010).

- Impact Per Publication (IPP): Desarrollado por el Centre forScience and TechnologyStudies (CWTS) de la universidad de Leiden, su valor se obtiene dividiendo el número de citas recibidas por los artículos publicados en una revista durante los tres años anteriores entre el número de artículos publicados en esa revista durante esos tres años (Elsevier, 2015).

- SourceNormalizedImpact per Paper (SNIP): Introducido por HenkMoed en 2010, es el IPP normalizado teniendo en cuenta las características y diferencias que existen entre los campos científicos como la frecuencia con la que se citan los autores, la rapidez de maduración del impacto de las citas y el grado de cobertura de la base de datos para un campo concreto (Moed 2010).

- Índice H: Propuesto por el físico Hirsch se define como el número $h$ de artículos con un número de citas $\geq h$ (Hirsch, 2005). Este índice combina tanto la producción como el impacto (Leydesdorff, 2009) y, aunque inicialmente se desarrolló para medir la producción individual de los investigadores, enseguida se aplicó también a las revistas como un complemento de los factores de impacto de éstas (Braun; Glänzel; Schubert, 2006). El índice H puede ser calculado con datos de WoS, Scopus o Google Scholar.

La existencia de tantos indicadores requiere conocer cómo y en qué grado se relacionan unos con otros y son muchos los estudios que se han llevado a cabo para analizar la correlación entre ellos. Algunos se han centrado en comparar el 
número de citas recibidas por investigadores (Bar-Ilan, 2008;Meho; Rogers, 2008) o por revistas en determinadas áreas de conocimiento o especialidades (LópezIllescas; Moya-Anegón; Moed, 2009), incluso se han comparado los países según el número de artículos y de citas recibidas (Archambault; Campbell; Gingras; Larivière, 2009).

En general, los diversos estudios realizados se caracterizan por analizar la correlación entre los indicadores:

- Utilizando muestras pequeñas de revistas de WoS y analizando unos pocos indicadores(Davis, 2008; Vanclay, 2008; Bador; Lafouge, 2009; Rizkallah; Sin, 2010).

- Utilizando todas las revistas de WoS y Scopus pero sin discriminar en las grandes áreas de Ciencias y Ciencias Sociales ni analizar todos los indicadores disponibles (Leydesdorff, 2009; Elkins; Maher; Herbert; Moseley; Sherrington, 2010; Torres-Salinas; Jiménez-Contreras, 2010).

- En revistas procedentes de ambas bases de datos pero en áreas de conocimiento específicas y solo algunos indicadores: el FI y el SJR en revistas de educación (Chou, 2013), el índice H y el FI en revistas españolas de biomedicina (García-Pachón; Padilla-Navas, 2014) o en revistas de biología reproductiva (Han; Yu; Wang, 2010), el índice H y el FI 5 años en revistas de trabajo social (Hodge; Lacasse, 2011); el FI, Eigenfactor y SJR en revistas de obstetricia y ginecología (Jamali; Salehi-Marzijarani; Ayatollahi, 2014); el FI, SJR, Eigenfactor y AIS en 77 revistas de 10 campos diferentes (Rousseau, 2009); o el FI, el Eigenfactor y el índice $\mathrm{H}$ en revistas de ingeniería química (Yin, 2011).

- Empleando casi todos los indicadores pero en las revistas más importantes de una categoría específica como Rehabilitación (Franchignoni; Lasa, 2011) o en revistas de un país concreto como Corea (Kim; Huh; Chu, 2014).

Ninguno de ellos utiliza todos los indicadores bibliométricos ni estudian la correlación de manera global en todas las revistas coincidentes en las dos bases de datos más importantes, WoS y Scopus. Además, algunos emplean el coeficiente de correlación de Pearson y otros el de Spearman, lo que produce resultados dispares entre pares de indicadores.

En este contexto, el presente estudio aporta tres características diferenciadoras de los publicados hasta ahora:

- El número total de indicadores bibliométricos, ya que ninguno ha utilizado todos ellos

- El mayor número de revistas analizadas, y

- La comparación de los valores obtenidos con el coeficiente de correlación de Spearman y de Pearson 
El objetivo de este estudio no es averiguar qué indicadores son mejores o más adecuados para determinar la importancia de la revista medida a través de su impacto en la comunidad científica, sino analizar la correlación existente entre ellos. Ya que todos los indicadores evalúan la popularidad y/o prestigio de las revistas, deberían presentar una alta correlación entre ellos a pesar de que utilizan diferentes fórmulas matemáticas. Por ello, la hipótesis es que algunos tienen un grado de correlación muy alto entre sí y, por tanto, son redundantes desde un punto de vista estadístico.

De manera más específica, se pretende estudiar la correlación en todas las revistas coincidentes en WoS y Scopus, tanto de manera global como en las dos grandes áreas de ciencias y ciencias sociales para determinar posibles diferencias entre ellas, así como analizar posibles diferencias en los valores de los coeficientes de correlación de Pearson y Spearman.

\section{MÉTODO}

La población estuvo formada por todas las revistas del Science Citation Index y del Social Science Citation Index indizadas en WoS y que, a su vez, estaban incluidas en la base de datos Scopus.

Para ello, se descargaron las revistas con los indicadores correspondientes al año 2013 (último año calculado y accesible del JCR) de las siguientes bases de datos durante la última semana del mes de marzo de 2015:

- Base de datos Web of Science: Desde la herramienta de análisis interactivo del JCR ${ }^{\circledR}$ (https://www.accesowok.fecyt.es/factor/getJCR.php) el Factor de impacto, Factor de impacto 5 años, índice de inmediatez, Eigenfactor, Influencia del artículo. El número total de revistas, una vez eliminadas las duplicadas, fue de 11.022.

- Base de datos Scopus a través de SCImago Journal\& Country Rank (http://www.scimagojr.com/journalrank.php): SJR, índice H, citas recibidas/ documentos publicados en los dos años anteriores (FI2Scopus). El número total de revistas fue de 29.385 .

- Base de datos Scopus a través de Journal Metrics (http://www.journalmetrics.com/): los indicadores SJR, SNIP e IPP. El número total de revistas fue de 34.130

Se creó una base de datos relacional con los indicadores bibliométricos mencionados de todas las revistas de WoS incluidas en Scopus.

Se identificaron y eliminaron del fichero las revistas duplicadas por nombre y/o ISSN, quedando al final un total de 10.700 revistas de WoS (el 97\%) incluidas en Scopus. 
Posteriormente se exportaron los datos al SPSS v.22, desde donde se realizó el análisis estadístico. Para estudiar la correlación entre los diversos indicadores se calculó el coeficiente de correlación de Spearman, tanto de manera general en todas las revistas y separado por áreas de conocimiento, Ciencias (revistas de Science Citation Index) y Ciencias Sociales (Social Science Citation Index), calculando también el coeficiente de correlación de Pearson para analizar las posibles diferencias.Aquellas revistas del JCR clasificadas tanto en el área de Ciencias como en Ciencias Sociales, se incluyeron solo en el campo de Ciencias, dejando en el Ciencias Sociales aquellas exclusivas de esta área de conocimiento. En las matrices de correlaciones se utilizó la opción listwisepara que el número de revistas fuera siempre el mismo entre cada pareja de indicadores, siendo 9.657 las revistas que contenían valores en todos los indicadores.

\section{RESULTADOS}

\subsection{ESTADÍSTICA DESCRIPTIVA}

La estadística descriptiva de los indicadores muestra una gran dispersión de los datos y una distribución fuertemente asimétrica a la derecha y muy leptocúrtica en todos ellos (tabla 1). El test de Kolmogorov-Smirnov confirma que ningún indicador sigue una distribución normal, condición indispensable para elegir el coeficiente de correlación de Pearson, por lo que para analizar la relación entre los indicadores se ha aplicado el coeficiente de correlación no paramétrico de Spearman.

Tabla 1. Estadística descriptiva de los indicadores bibliométricos.

\begin{tabular}{|l|c|c|c|c|c|c|}
\hline Indicador & $\mathrm{N}$ & Media (DT) & Mediana (RI) & Curtosis & Asimetría & $\begin{array}{c}\text { Test de K-S } \\
(\text { valor } \mathrm{p})\end{array}$ \\
\hline FI & 10.659 & $1,92(3,14)$ & $1,22(1,66)$ & 679,93 & 17,05 & $<0,001$ \\
\hline FI5 & 10.013 & $2,18(3,19)$ & $1,47(1,85)$ & 175,63 & 9,38 & $<0,001$ \\
\hline INME & 10.444 & $0,41(0,73)$ & $0,24(0,40)$ & 251,85 & 10,92 & $<0,001$ \\
\hline EF & 10.695 & $0,0091(0,03)$ & $0,0021(0,005)$ & 686,17 & 22,08 & $<0,001$ \\
\hline AIS & 10.013 & $0,81(1,43)$ & $0,51(0,64)$ & 117,22 & 8,71 & $<0,001$ \\
\hline SJR & 10.576 & $1,15(1,76)$ & $0,73(0,90)$ & 109,41 & 8,10 & $<0,001$ \\
\hline FI2SC & 10.589 & $2,02(2,72)$ & $1,39(1,78)$ & 302,45 & 10,97 & $<0,001$ \\
\hline SNIP & 10.496 & $1,19(1,19)$ & $0,99(0,73)$ & 1179,57 & 22,41 & $<0,001$ \\
\hline IPP & 10.496 & $1,96(2,88)$ & $1,35(1,68)$ & 868,37 & 19,13 & $<0,001$ \\
\hline H & 10.589 & $42,87(43,50)$ & $31(39)$ & 38,46 & 4,12 & $<0,001$ \\
\hline
\end{tabular}

(DT=Desviación típica; RI= Rango intercuartílico; Test de K-S= Test de Kolmogorov-Smirnov corrección de Lilliefors)

FI: Factor de Impacto de JCR; FI5: Factor de Impacto 5 años de JCR; INME: índice de Inmediatez; EF: Eigenfactor; AIS: ArticleInfluence Score; SJR: ScimagoJournal Rank; FI2SC: Factor de Impacto de 2 años creado de Scopus; SNIP: SourceNormalizedImpact per Paper; IPP: Impact per Publication; H: índice H de Scopus. 


\subsection{CORRELACIONES ENTRE LOS INDICADORES}

Se presentan los resultados de manera global y por grandes áreas temáticas para comprobar si hay diferencias, que pueden ser debidas entre otros factores a distintos patrones o hábitos de citación.

La tabla 2 presenta la matriz de correlación de los indicadores en la que puede observarse un grado de correlación muy alto $(>0,94)$ entre el Factor de Impacto, FI de 5 años, FI de Scopus y el IPP. Es lógico que presenten estos valores tan altos ya que todos estos indicadores dividen las citas que recibe una revista durante un periodo de tiempo específico por el número de artículos publicados por la revista en el mismo periodo, diferenciándose principalmente por el número de años analizados. La ampliación de la ventana de citación, que pretende favorecer a las áreas en las que se tarda más tiempo en citar como aquellas que pertenecen a las ciencias sociales, tampoco tiene una influencia importante en los valores, tal y como se ve en la tabla 3, en la que los coeficientes de correlación apenas varían en las áreas de Ciencias y de Ciencias Sociales.

Tabla 2. Coeficientes de correlación (rho de Spearman) entre cada par de indicadores.

( $\mathrm{N}^{\mathrm{o}}$ de revistas: 9657$)$

\begin{tabular}{|c|c|c|c|c|c|c|c|c|c|c|c|}
\hline & \multicolumn{5}{|c|}{ WoS } & \multicolumn{5}{|c|}{ Scopus } \\
\hline & & FI & FI5 & INME & $\mathrm{EF}$ & AIS & SJR & FI2SC & SNIP & IPP & $\mathrm{H}$ \\
\hline \multirow{5}{*}{3} & FI & 1 & & & & & & & & & \\
\hline & FI5 & 0,968 & 1 & & & & & & & & \\
\hline & INME & 0,799 & 0,776 & 1 & & & & & & & \\
\hline & $\mathrm{EF}$ & 0,800 & 0,807 & 0,676 & 1 & & & & & & \\
\hline & AIS & 0,834 & 0,891 & 0,681 & 0,780 & 1 & & & & & \\
\hline \multirow{5}{*}{ 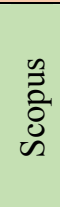 } & SJR & 0,839 & 0,874 & 0,668 & 0,792 & 0,923 & 1 & & & & \\
\hline & FI2SC & 0,963 & 0,948 & 0,750 & 0,783 & 0,825 & 0,865 & 1 & & & \\
\hline & SNIP & 0,645 & 0,690 & 0,489 & 0,618 & 0,743 & 0,808 & 0,717 & 1 & & \\
\hline & IPP & 0,955 & 0,968 & 0,749 & 0,798 & 0,851 & 0,891 & 0,977 & 0,738 & 1 & \\
\hline & $\mathrm{H}$ & 0,763 & 0,785 & 0,637 & 0,869 & 0,707 & 0,732 & 0,771 & 0,595 & 0,788 & 1 \\
\hline
\end{tabular}

También tiene una correlación muy alta el SJR con el AIS $(0,923)$, algo nada sorprendente porque en sus fórmulas intervienen variables comunes y no utilizadas por los factores de impacto, como la limitación del número o exclusión de las autocitas y el peso que se otorga a las revistas citantes según su prestigio o importancia. Al analizar las revistas por áreas de conocimiento, se observa una leve diferencia entre el área de Ciencias $(0,930)$ y de Ciencias Sociales $(0,896)$. EL SJR también tiene una correlación muy alta con el Factor de Impacto de 5 años $(0,874)$, muy similar en las dos áreas, a pesar de que este último indicador no pondera revistas ni excluye las autocitas.

El AIS a su vez también mantiene una alta correlación con el Factor de Impacto de 5 años $(0,891)$, con el que coincide en el periodo de años analizado, y algo 
menor con el Factor de Impacto de 2 años de JCR $(0,834)$ y de Scopus $(0,825)$, lo que invita a pensar que estas leves diferencias pueden ser debidas al número de años ya que no hay otras variables que puedan influir.

El comportamiento del AIS con el Eigenfactor es diferente según el área de conocimiento. En Ciencias mantiene el mismo valor que sin discriminar por áreas $(0,780)$, pero en Ciencias Sociales aumenta considerablemente $(0,891)$, revelando que el ajuste del Eigenfactor por el número de artículos publicados de la revista tiene mayor efecto en Ciencias Sociales y provoca una asociación más fuerte en este campo.

El índice $\mathrm{H}$ tiene mayor correlación con el Eigenfactor $(0,869)$, algo comprensible ya que ambos indicadores son los únicos que tienen en cuenta el número de citas recibidas sin normalizar por el número de artículos publicados.

Tabla 3. Coeficientes de correlación (rho de Spearman) entre cada par de indicadores en las áreas de Ciencias y de Ciencias Sociales. ( $\mathrm{N}^{\circ}$ de revistas en Ciencias: 7571. $\mathrm{N}^{\circ}$ de revistas en Ciencias Sociales: 2086)

\begin{tabular}{|c|c|c|c|c|c|c|c|c|c|c|c|}
\hline & \multicolumn{5}{|c|}{ WoS } & \multicolumn{5}{|c|}{ Scopus } \\
\hline & & FI & FI5 & INME & EF & AIS & SJR & FI2SC & SNIP & IPP & $\mathrm{H}$ \\
\hline \multirow{5}{*}{$\begin{array}{l}n \\
0 \\
3\end{array}$} & FI & 1 & & & & & & & & & \\
\hline & FI5 & $\begin{array}{l}0,974 \\
0,952\end{array}$ & 1 & & & & & & & & \\
\hline & INME & $\begin{array}{l}0,828 \\
0,661\end{array}$ & $\begin{array}{l}0,807 \\
0,640\end{array}$ & 1 & & & & & & & \\
\hline & $\mathrm{EF}$ & $\begin{array}{l}0,778 \\
0,815\end{array}$ & $\begin{array}{l}0,788 \\
0,864\end{array}$ & $\begin{array}{l}0,686 \\
0,572\end{array}$ & 1 & & & & & & \\
\hline & AIS & $\begin{array}{l}0,858 \\
0,847\end{array}$ & $\begin{array}{l}0,899 \\
0,907\end{array}$ & $\begin{array}{l}0,725 \\
0,570\end{array}$ & $\begin{array}{l}0,780 \\
0,891\end{array}$ & 1 & & & & & \\
\hline \multirow{5}{*}{ 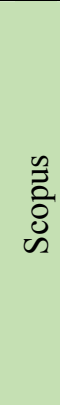 } & SJR & $\begin{array}{l}0,858 \\
0,853\end{array}$ & $\begin{array}{l}0,880 \\
0,882\end{array}$ & $\begin{array}{l}0,713 \\
0,545\end{array}$ & $\begin{array}{l}0,801 \\
0,852\end{array}$ & $\begin{array}{l}0,930 \\
0,896\end{array}$ & 1 & & & & \\
\hline & FI2SC & $\begin{array}{l}0,963 \\
0,953\end{array}$ & $\begin{array}{l}0,951 \\
0,934\end{array}$ & $\begin{array}{l}0,777 \\
0,609\end{array}$ & $\begin{array}{l}0,760 \\
0,811\end{array}$ & $\begin{array}{l}0,845 \\
0,830\end{array}$ & $\begin{array}{l}0,880 \\
0,885\end{array}$ & 1 & & & \\
\hline & SNIP & $\begin{array}{l}0,668 \\
0,730\end{array}$ & $\begin{array}{l}0,703 \\
0,744\end{array}$ & $\begin{array}{l}0,527 \\
0,464\end{array}$ & $\begin{array}{l}0,646 \\
0,716\end{array}$ & $\begin{array}{l}0,738 \\
0,779\end{array}$ & $\begin{array}{l}0,806 \\
0,835\end{array}$ & $\begin{array}{l}0,742 \\
0,783\end{array}$ & 1 & & \\
\hline & IPP & $\begin{array}{l}0,961 \\
0,926\end{array}$ & $\begin{array}{l}0,970 \\
0,953\end{array}$ & $\begin{array}{l}0,781 \\
0,594\end{array}$ & $\begin{array}{l}0,779 \\
0,831\end{array}$ & $\begin{array}{l}0,866 \\
0,853\end{array}$ & $\begin{array}{l}0,900 \\
0,909\end{array}$ & $\begin{array}{l}0,980 \\
0,965\end{array}$ & $\begin{array}{l}0,754 \\
0,805\end{array}$ & 1 & \\
\hline & $\mathrm{H}$ & $\begin{array}{l}0,748 \\
0,747\end{array}$ & $\begin{array}{l}0,770 \\
0,802\end{array}$ & $\begin{array}{l}0,651 \\
0,521\end{array}$ & $\begin{array}{l}0,869 \\
0,850\end{array}$ & $\begin{array}{l}0,709 \\
0,747\end{array}$ & $\begin{array}{l}0,739 \\
0,747\end{array}$ & $\begin{array}{l}0,756 \\
0,766\end{array}$ & $\begin{array}{l}0,618 \\
0,642\end{array}$ & $\begin{array}{l}0,772 \\
0,797\end{array}$ & 1 \\
\hline
\end{tabular}

Los indicadores con un comportamiento marcadamente diferente para las revistas de Science Citation Index y Social Science Citation Index son los siguientes:

- El índice de Inmediatez tiene un coeficiente de correlación sensiblemente inferior en el campo de las Ciencias Sociales con el resto de indicadores lo 
que evidencia que las revistas con mayor factor de impacto en este campo no suelen tener un impacto inmediato tan alto.

- El Eigenfactor tiene una correlación con valores ligeramente superiores en el campo de Ciencias Sociales con todos los demás indicadores, salvo el índice $\mathrm{H}$. Ello indica que esta medida favorece a las revistas de este campo.

- EL SNIP también tiene una correlación ligeramente superior en Ciencias Sociales con todos los indicadores menos con el índice de inmediatez, lo que evidencia también que las revistas de esta área se ven favorecidas.

Los indicadores de impacto (FI, FI5, SJR y FI2SC) apenas varían en las dos áreas.

\section{DISCUSIÓN}

En la tabla 4 se presentan los resultados obtenidos en nuestro estudiojunto con aquellos más similares encontrados en la literatura. Todos ellos comparten la característica de que utilizan indicadores obtenidos de las dos bases de datos, WoS y Scopus. Los trabajos de Leydesdorff(2009) y Elkins (2010) aplican solo tres indicadores aunque de manera global sin discriminar campos temáticos. En el de Torres (2010) se han seleccionado las revistas de los campos de medicina (med) y de ciencias sociales (ccss), ya que no se presentan los coeficientes de correlación de manera global. Los trabajos de Kim (2014) y Rousseau (2009) se han seleccionado por utilizar un número amplio de indicadores, si bien en subconjuntos con un número de revistas mucho más reducido.

Se indica en cada uno de ellos el número de revistas analizadas, el coeficiente de correlación utilizado y si la matriz de correlacionestiene el mismo número de revistas en todos los coeficientes calculados (matriz list) o varía según los datos disponibles en cada pareja de indicadores (matriz pair). 
Tabla 4. Comparación de coeficientes de correlación del estudio actual con otros trabajos

\begin{tabular}{|c|c|c|c|c|c|c|c|}
\hline & $\begin{array}{c}\text { Estudio } \\
(\mathbf{2 0 1 5 )} \\
\mathrm{n}=9657 \\
\rho \text { Spearman } \\
\text { matriz list } \\
\end{array}$ & $\begin{array}{c}\text { Leydesdorff } \\
\mathbf{( 2 0 0 9 )} \\
\mathrm{n} \approx 6100 \\
\text { r de Pearson } \\
\text { matriz pair } \\
\end{array}$ & 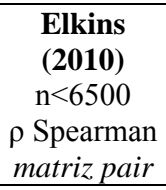 & \begin{tabular}{|c|} 
Torres \\
(2010) med \\
$\mathrm{n}=1949$ \\
$\mathrm{r}$ de Pearson \\
matriz list \\
\end{tabular} & $\begin{array}{c}\text { Torres } \\
\text { (2010) ccss } \\
\mathrm{n}=1017 \\
\text { r de Pearson } \\
\text { matriz list } \\
\end{array}$ & $\begin{array}{c}\text { Kim } \\
\mathbf{( 2 0 1 4 )} \\
\mathrm{n}=62 \\
\text { r de Pearson } \\
\text { matriz list }\end{array}$ & \begin{tabular}{|c|} 
Rousseau \\
$\mathbf{( 2 0 0 9 )}$ \\
$\mathrm{n}=77$ \\
$\mathrm{r}$ de Pearson \\
matriz list \\
\end{tabular} \\
\hline FI-INME & 0,799 & 0,877 & & & & & \\
\hline FI-SJR & 0,839 & 0,796 & 0,89 & 0,8342 & 0,4753 & 0,429 & 0,905 \\
\hline FI-AIS & 0,834 & & 0,79 & 0,9352 & 0,1044 & 0,883 & 0,918 \\
\hline FI-FI5 & 0,968 & & & & & 0,974 & \\
\hline FI-EF & 0,800 & & & 0,5818 & 0,5527 & 0,668 & 0,827 \\
\hline FI-H & 0,763 & & & & & 0,435 & 0,869 \\
\hline FI-SNIP & 0,645 & & & 0,8660 & 0,6115 & & \\
\hline AIS-SJR & 0,923 & & 0,61 & 0,8895 & 0,1584 & 0,345 & 0,813 \\
\hline AIS-FI5 & 0,891 & & & & & 0,937 & \\
\hline AIS-EF & 0,780 & & & 0,5674 & 0,0256 & 0,684 & 0,827 \\
\hline AIS-H & 0,707 & & & & & 0,200 & 0,855 \\
\hline AIS-SNIP & 0,743 & & & 0,7811 & 0,0610 & & \\
\hline EF-FI5 & 0,807 & & & & & 0,759 & \\
\hline EF-SJR & 0,792 & & & 0,4551 & 0,5063 & $-0,095$ & 0,731 \\
\hline EF-H & 0,869 & & & & & & 0,951 \\
\hline EF-SNIP & 0,618 & & & 0,4002 & 0,5041 & & \\
\hline SJR-SNIP & 0,808 & & & 0,7423 & 0,3904 & & \\
\hline SJR-H & 0,732 & & & & & 0,475 & 0,760 \\
\hline SJR-INME & 0,668 & 0,671 & & & & & \\
\hline
\end{tabular}

El único estudio que utiliza el coeficiente de correlación de Spearman es el de Elkins, el resto utilizan el de Pearson. A este respecto, es preciso destacar las diferencias que se producen al utilizar uno u otro y que pueden verse en la tabla 5, afectando de manera muy notable al Eigenfactor, que obtiene valores mucho más bajos con el coeficiente de Pearson, y de manera más leve al índice $\mathrm{H}$, con valores más bajos en el de Pearson, y SNIP,siendo en este caso algo inferiores en el de Spearman con algunos indicadores.

Este comportamiento distinto del coeficiente de correlación paramétrico y no paramétrico explica las discrepancias tan severas observadas entre los valores obtenidos con el Eigenfactor en nuestro estudio y el de Torres; estas diferencias son más leves cuando se comparan con los estudios de Kim y Rousseau, debido probablemente al número más reducido de revistas analizadas. 
Tabla 5. Diferencias en los valores de los coeficientes de correlación de Spearman y de Pearson entre los indicadores bibliométricos. Orden en la tabla ( $\left.\begin{array}{c}\text { Spearman } \\ \text { Pearson }\end{array}\right)$

\begin{tabular}{|c|c|c|c|c|c|c|c|c|c|c|c|}
\hline & \multicolumn{5}{|c|}{ WoS } & \multicolumn{5}{|c|}{ Scopus } \\
\hline & & FI & FI5 & INME & EF & AIS & SJR & FI2SC & SNIP & IPP & $\mathrm{H}$ \\
\hline \multirow{5}{*}{$\begin{array}{l}n \\
0 \\
3\end{array}$} & FI & 1 & & & & & & & & & \\
\hline & FI5 & $\begin{array}{l}0,968 \\
0,966\end{array}$ & 1 & & & & & & & & \\
\hline & INME & $\begin{array}{l}0,799 \\
0,856\end{array}$ & $\begin{array}{l}0,776 \\
0,833\end{array}$ & 1 & & & & & & & \\
\hline & $\mathrm{EF}$ & $\begin{array}{l}0,800 \\
0,407\end{array}$ & $\begin{array}{l}0,807 \\
0,420\end{array}$ & $\begin{array}{l}0,676 \\
0,392\end{array}$ & 1 & & & & & & \\
\hline & AIS & $\begin{array}{l}0,834 \\
0,869\end{array}$ & $\begin{array}{l}0,891 \\
0,927\end{array}$ & $\begin{array}{l}0,681 \\
0,760\end{array}$ & $\begin{array}{l}0,780 \\
0,411\end{array}$ & 1 & & & & & \\
\hline \multirow{5}{*}{$\begin{array}{l}0 \\
\tilde{\delta} \\
\tilde{D} \\
\end{array}$} & SJR & $\begin{array}{l}0,839 \\
0,829\end{array}$ & $\begin{array}{l}0,874 \\
0,875\end{array}$ & $\begin{array}{l}0,668 \\
0,699\end{array}$ & $\begin{array}{l}0,792 \\
0,395\end{array}$ & $\begin{array}{l}0,923 \\
0,937\end{array}$ & 1 & & & & \\
\hline & FI2SC & $\begin{array}{l}0,963 \\
0,962\end{array}$ & $\begin{array}{l}0,948 \\
0,955\end{array}$ & $\begin{array}{l}0,750 \\
0,802\end{array}$ & $\begin{array}{l}0,783 \\
0,376\end{array}$ & $\begin{array}{l}0,825 \\
0,855\end{array}$ & $\begin{array}{l}0,865 \\
0,863\end{array}$ & 1 & & & \\
\hline & SNIP & $\begin{array}{l}0,645 \\
0,780\end{array}$ & $\begin{array}{l}0,690 \\
0,754\end{array}$ & $\begin{array}{l}0,489 \\
0,634\end{array}$ & $\begin{array}{l}0,618 \\
0,233\end{array}$ & $\begin{array}{l}0,743 \\
0,718\end{array}$ & $\begin{array}{l}0,808 \\
0,729\end{array}$ & $\begin{array}{l}0,717 \\
0,791\end{array}$ & 1 & & \\
\hline & IPP & $\begin{array}{l}0,955 \\
0,973\end{array}$ & $\begin{array}{l}0,968 \\
0,951\end{array}$ & $\begin{array}{l}0,749 \\
0,813\end{array}$ & $\begin{array}{l}0,798 \\
0,375\end{array}$ & $\begin{array}{l}0,851 \\
0,851\end{array}$ & $\begin{array}{l}0,891 \\
0,853\end{array}$ & $\begin{array}{l}0,977 \\
0,968\end{array}$ & $\begin{array}{l}0,738 \\
0,841\end{array}$ & 1 & \\
\hline & $\mathrm{H}$ & $\begin{array}{l}0,763 \\
0,604\end{array}$ & $\begin{array}{l}0,785 \\
0,649\end{array}$ & $\begin{array}{l}0,637 \\
0,558\end{array}$ & $\begin{array}{l}0,869 \\
0,678\end{array}$ & $\begin{array}{l}0,707 \\
0,591\end{array}$ & $\begin{array}{l}0,732 \\
0,613\end{array}$ & $\begin{array}{l}0,771 \\
0,631\end{array}$ & $\begin{array}{l}0,595 \\
0,432\end{array}$ & $\begin{array}{l}0,788 \\
0,604\end{array}$ & 1 \\
\hline
\end{tabular}

A pesar de las diferencias debidas al coeficiente de correlación utilizado en los estudios y que limitan su comparación, se exponen a continuación lo más destacado.

- Factores de Impacto: El FI, FI5, AIS y SJR miden el impacto medio de citas por artículo, y por lo tanto, deberían ser similares independientemente del tamaño de la revista, mientras que el EF mide el impacto total de las citas, que depende del tamaño de la revista.

Los valores más altos de correlación se obtienen entre el FI y el FI5, coincidiendo con los resultados de Kim (2014). Una correlación tan alta indica que prácticamente están midiendo lo mismo y que la ampliación de la ventana de citación de 2 a 5 años no influye en los valores obtenidos.

En general, el FI correlaciona bien con los demás indicadores, obteniendo los valores más bajos con el SNIP, tanto en nuestro estudio como en el de Torres en las revistas de ciencias sociales.

La correlación entre el FI y el índice $\mathrm{H}$ en los estudios representados en la tabla4 muestra mayor variabilidad, probablemente debidaal conjunto de revistas utilizado en cada uno de ellos; así por ejemplo, se han encontrado valores altos de 0,88 en revistas de psiquiatría (Bador; Lafouge, 2011), de 0,77 en revistas de 
toxicología (Bird, 2008) y de 0,64 en revistas de biología reproductiva (Han; Yu; Wang, 2010).El índice $\mathrm{H}$ depende del número de años de la revista y del número de artículos publicados, mientras que los factores de impacto del periodo de citación y publicación.

También se hallaron correlaciones altas (mayores de 0,85) del SJR con FI, FI5años y FI2, con un comportamiento distinto en los demás estudios, alcanzando valores muy bajos en los de Torres y Kim $(\mathrm{r}=0,47$ y $\mathrm{r}=0,42)$. Valores muy altos se han encontrado también en el estudio deChou (2013) en revistas de educación $(\mathrm{r}=0,91)$, sin que el tipo de indicador influyera en el rango de las revistas analizadas. Coeficientes muy altos del SJR con los diversos factores de impacto puede indicar que las autocitas no sean un factor de confusión importante y que dar más peso a las citas de revistas de calidad no altera materialmente los resultados (Rocha-e-Silva, 2010).

- Influencia del Artículo: El AIS también correlaciona muy bien con todos los factores de impacto obteniendo en nuestro estudiolos valores más altos con el SJR. En el resto de estudios presenta valores muy dispares difícilmente explicables por usar un coeficiente diferente, un subconjunto pequeño de revistas o un campo temático diferente.

También presenta valores altos con el FI5, en consonancia con los resultados obtenidos por Kim (2014) y Yin (2011) en revistas de ingeniería química $(\mathrm{r}=0,9626)$, algo que no debe sorprender ya que son medidas directamente comparables (Bergstrom, 2007); con el FI presenta también unos valores altos en nuestro estudio $(0,83)$ aunque no se comporta de la misma manera en todos los demás, si bien se ha considerado como una alternativa viable al FI con la ventaja de que es una fuente de acceso abierto (Oosthuizen; Fenton, 2013), y a pesar de que se calculan de manera diferente produce un rango similar al obtenido con el FI (Rizkallah; Sin, 2010).

- Eigenfactor: Es el indicador que presenta mayores diferencias en los valores obtenidos por el coeficiente de correlación de Pearson y de Spearman, por lo que no es comparable con ninguno de los estudios de la Tabla 4 ya que el único que utiliza el de Spearman, no lo aplica a este indicador y el resto de estudios utilizan el paramétrico. Estas diferencias tan grandes provocan que en los estudios que han utilizado el coeficiente de Pearson los valores sean muy bajos y en alguno se haya concluido que no tiene correlación con el SJR (Jamali; Salehi-Marzijarani; Ayatollahi, 2014).

Se ha hallado una correlación alta con los indicadores de impacto, similar al obtenido por Davis $(2008)$ con el FI en revistas de medicina $(r h o=0,84)$ en cuyo estudio no se producía una variación drástica en el orden de las revistas según estos indicadores. La correlación más alta la obtiene con el índice $\mathrm{H}$ (superior a 0,85 en las dos áreas), y parece razonable que sea así ya que miden el impacto total de las citas (que depende del tamaño de la revista) y comparten la misma 
característica de no estar normalizados por el número de artículos publicados en las revistas, en consonancia con el valor encontrado por Yin (2011) de 0,9139.

Aunque en nuestro estudio ha mostrado una alta correlación con los indicadores de impacto, ha sido mayor en el área de ciencias sociales que en ciencias, en consonancia con los resultados obtenidos por Franceschet (2010).

Índice H: También obtiene unos valores diferentes según el coeficiente de correlación utilizado, siendo mayores con el de Spearman. En general, las correlaciones con los indicadores de impacto ofrecen unos valores no muy altos (alrededor de 0,74 en todos ellos), lo que indica que miden diferentes dimensiones y ofrecen diferente información aunque complementaria; los factores de impacto miden la influencia de la revista en un plazo más corto que el índice $\mathrm{H}$, que mide la influencia de la revista a lo largo de toda su vida, de ahí que en diversos estudios la clasificación de las revistas sea diferente (García-Pachón; PadillaNavas, 2014).Se ha señalado en la literatura que el índice $H$ se correlaciona más con la valoración de la calidad de las revistas por expertos que el FI 5 años, por lo que este indicador puede tener utilidad para medir la calidad de las revistas (Hodge; Lacasse, 2011)

- IPP, SNIP: El Impacto Por Publicación (IPP) presenta unos valores muy altos (superiores a 0,95 ) con los indicadores de impacto (FI, FI5, FI2), algo lógico ya que la principal diferencia entre ellos es la ventana de citación utilizada. La correlación baja sensiblemente cuando se normaliza (SNIP) y se tienen en cuenta características propias de cada área y la cobertura de bases de datos, alcanzando los valores más altos con el SJR, indicador creado con la misma base de datos.

\section{Limitaciones}

Se han analizado indicadores calculados con datos procedentes de dos bases diferentes, WoS y Scopus; al seleccionar solamente aquellas revistas que coinciden en ambas bases de datos, el número de artículos es el mismo, si bien difiere el número de citas que reciben, aunque hay estudios donde se ha evidenciado que los indicadores son independientes de la base de datos (Archambault; Campbell; Gingras; Larivière, 2009).

\section{CONCLUSIONES}

Los indicadores de popularidad (factores de impacto e IPP) tienen una correlación muy alta entre sí $(>0,95)$, lo que indica que factores como el periodo de tiempo utilizado en sus fórmulas, pertenecer al área de ciencias o ciencias sociales, $o$ la base de datos utilizada para las citas noinfluyen en el orden de las revistas.

Los indicadores basados en el prestigio de las revistas tienen un comportamiento diferente. El AIS y el SJR, que están normalizados por el número de artículos publicados en las revistas y miden, por tanto, el impacto medio por artículo, tienen una correlación muy alta entre sí, sobre todo en el área de ciencias, 
y también con el FI5, mientras que el Eigenfactor tiene mayor correlación con el índice $\mathrm{H}$, algo lógico al ser indicadores que miden el impacto global.

EL SJR también muestra una correlación muy alta en ambas áreas con el IPP, coincidiendo con la ventana de citación de 3 años y que utilizan la misma base de datos, pero no parece que otras variables incluidas en la fórmula del SJR influyan mucho en el rango de las revistas.

Correlaciones muy altas indican que miden propiedades similares de las revistas científicas y, por tanto, hay evidencia de validez convergente entre los siguientes indicadores al existir una correlación muy alta entre ellos: a) todoslos factores de impacto entre ellos mismos (FI, FI5, FI2Scopus e IPP), b) AIS con SJR y FI5, y c) SJR con IPP.

$\mathrm{El}$ índice $\mathrm{H}$ tiene una correlación moderada con los indicadores de impacto y de prestigio $(\approx 0,70)$, lo que indica que miden dimensiones diferentes, aportan nueva información y, por tanto, pueden ser complementarios.

El comportamiento de los indicadores estudiados es muy similar en las dos grandes áreas, ciencias y ciencias sociales.

Se ha señalado en la literatura que la calidad de una revista, medida a través de indicadores de popularidad y prestigio, es un concepto multidimensional en el que pueden intervenir varios factores como hábitos de citación, tamaño de la comunidad científica, tiempo, etc. La altísima correlación encontrada entre diversos indicadores sugiere que recogen la misma información y que las diferencias en sus fórmulas no afectan al rango de las revistas, por lo que habría que plantearse la necesidad de tantos indicadores diferentes al medir realmente lo mismo, ya que no añaden información nueva.

\section{BIBLIOGRAFÍA}

ALTHOUSE, B.M.; WEST, J.D.; BERGSTROM, C.T.; BERGSTROM, T. (2009). "Differences in impact factor across fields and over time". Journal of the American Society for Information Science and Technology, vol. 60, $\mathrm{n}^{\circ} 1$, pp. 27-34. DOI 10.1002/asi.20936.

ARCHAMBAULT, É.; CAMPBELL, D.; GINGRAS, Y.; LARIVIÈRE, V. (2009). "Comparing bibliometric statistics obtained from the web of science and Scopus". Journal of the American Society for Information Science and Technology, vol. 60, $\mathrm{n}^{\mathrm{0}}$ 7, pp. 1320-1326. DOI 10.1002/asi.21062.

BADOR, P.; LAFOUGE, T. (2009).“Comparative analysis between impact factor and h-index for pharmacology and psychiatry journals".Scientometrics, vol. 84, $\mathrm{n}^{\mathrm{o}}$ 1, pp. 65-79. DOI 10.1007/s11192-009-0058-2.

BADOR, P.; LAFOUGE, T. (2011).“Analyse comparative du facteurd'impactet de l'indice $h$ dans les revues de psychiatrie". Canadian Journal of Information and Library Science, vol. 35, $\mathrm{n}^{\circ}$ 2, pp. 109-121. 
BAR-ILAN, J. (2008). "Which h-index?- A comparison of WoS, Scopus and Google Scholar".Scientometrics, vol. 74, $\mathrm{n}^{\mathrm{o}}$ 2, pp. 257-271. DOI 10.1007/s11192-008-0216-y.

BERGSTROM, C. (2007). "Measuring the value and prestige of scholarly journals".College and Research Library News, vol. 68, $\mathrm{n}^{\mathrm{o}}$ 5, pp. 314316. $<$ http://crln.acrl.org/content/68/5/314.full.pdf $\geq$ [consulta14/06/2015]

BERGSTROM, C.T.; WEST, J.D.; WISEMAN, M. (2008). "The Eigenfactor metrics".The Journal of Neuroscience, vol. 28, $\mathrm{n}^{\circ}$ 45, pp. 11433-11434. DOI 10.1523/JNEUROSCI.0003-08.2008

BIRD, S.B. (2008).“Journal impact factors, h indices, and citation analyses in toxicology". Journal of Medical Toxicology, vol. 4, $\mathrm{n}^{\mathrm{o}}$ 4, pp. 261-274.DOI: $10.1007 / \mathrm{BF} 03161211$

BOLLEN, J.;c, R. (2009).“A principal component analysis of 39 scientific impact measures”.PLOS ONE, vol. $4, \quad \mathrm{n}^{\mathrm{o}} \quad 6, \quad \mathrm{pp}$. e6022.DOI:10.1371/journal.pone.0006022

BORDONS, M.; FERNANDEZ, M.T.; GOMEZ, I. (2002)."Advantages and limitations in the use of impact factor measures for the assessment of research performance in a peripheral country". Scientometrics, vol. 53, $\mathrm{n}^{\circ}$ 2, pp. 195-206.

BRAUN, T.; GLÄNZEL, W.; SCHUBERT, A. (2006). "A Hirsch-type index for journals".Scientometrics, vol. 69, $\mathrm{n}^{\circ} 1$, pp. 169-173. DOI:10.1007/s11192-006$0147-4$

CHOU, P. (2013). “An Exploratory Study of Database Evaluation Metrics : Comparison between IF and SJR".International Journal of Computer and Information Technology, vol. 2, $\mathrm{n}^{\mathrm{o}}$ 1, pp. 25-28.

DAVIS, P.M. (2008). "Eigenfactor: Does the principle of repeated improvement result in better estimates than raw citation counts?."Journal of the American Society for Information Science and Technology, vol. 59, n 13, pp. 2188-2188. DOI:10.1002/asi.20943

DONG, P.; LOH, M.; MONDRY, A. (2005).“The «impact factor» revisited”. Biomedical Digital Libraries, vol. 2, pp. 7. DOI:10.1186/1742-5581-2-7

DURIEUX, V.; GEVENOIS, P.A. (2010)."Bibliometric indicators: quality measurements of scientific publication". Radiology, vol. 255, $\mathrm{n}^{\circ}$ 2, pp. 342-351. DOI:10.1148/radiol.09090626

ELKINS, M.R.; MAHER, C.G.; HERBERT, R.D.; MOSELEY, A.M.; SHERRINGTON, C. (2010). "Correlation between the Journal Impact Factor and three other journal citation índices".Scientometrics, vol. 85, $\mathrm{n}^{\circ}$ 1, pp. 81-93. DOI:10.1007/s11192-010-0262-0

ELSEVIER, B.V. (2015). Journal metrics. $<$ http://www.journalmetrics.com/ipp.php $\geq$ [consulta 20/06/2015]

FERSHT, A. (2009). "The most influential journals: Impact Factor and Eigenfactor". Proceedings of the National Academy of Sciences of the United 
States of America, vol. 106, $\mathrm{n}^{\mathrm{o}}$ 17, pp. 6883-6884. DOI:10.1073/pnas.0903307106

FRANCHIGNONI, F.; LASA, S.M. (2011). "Bibliometric indicators and core journals in physical and rehabilitation medicine".Journal of Rehabilitation Medicine, vol. 43, nº 6, pp. 471-476. DOI:10.2340/16501977-0821

FRANCESCHET, M. (2010)."The difference between popularity and prestige in the sciences and in the social sciences: A bibliometric analysis". Journal of Informetrics, vol. 4, $\mathrm{n}^{\circ} 1$, pp. 55-63.

GARCÍA-PACHÓN, E.; PADILLA-NAVAS, I. (2014). "El factor de impacto y el índice $\mathrm{h}$ de las revistas biomédicas españolas". Medicina Clinica, vol. 142, $\mathrm{n}^{\circ}$ 5, pp. 226-227. DOI:10.1016/j.medcli.2013.09.014

GARFIELD, E. (1955). "Citation indexes to science: a new dimension in documentation through association of ideas". Science, vol. 122, no 3159, pp. 108-111. <http://garfield.library.upenn.edu/papers/science1955.pdf $\geq$ [consulta $10 / 05 / 2015]$

GARFIELD, E. (1972). "Citation analysis as a tool in journal evaluation”.Science, vol. 178, no 4060, pp. 471-479.

GARFIELD, E. (2006). "The history and meaning of the Journal Impact Factor".Journal of the American Medican Association, vol. 295, nº 1, pp. 9093.

GONZÁLEZ-PEREIRA, B.; GUERRERO-BOTE, V.P.; MOYA-ANEGÓN, F. (2010)."A new approach to the metric of journals scientific prestige: The SJR indicator". Journal of Informetrics, vol. 4, $\mathrm{n}^{\mathrm{o}}$ 3, pp. 379-391. DOI:10.1016/j.joi.2010.03.002

HAN, W.; YU, Q.; WANG, Y. (2010). "Comparative Analysis Between Impact Factor and h-Index for Reproduction Biology Journals". Journal of Animal and Veterinary Advances, vol. 9, $\mathrm{n}^{\mathrm{o}}$ 11, pp. 1552-1555. DOI:10.3923/javaa.2010.1552.1555

HARTER, S.P.; NISONGER, T.E. (1997).“ISI's Impact Factor as Misnomer: A Proposed New Measure to Assess Journal Impact". Journal of the American Society for Information Science, vol. 48, $\mathrm{n}^{\circ}$ 12, pp. 1146-1148.

HIRSCH, J.E. (2005). "An index to quantify an individual's scientific research output".Proceedings of the National Academy of Sciences of the United States of America, vol. 102, no 46, pp. 16569-16572. DOI:10.1073/pnas.0507655102

HODGE, D.R.; LACASSE, J.R. (2011)."Evaluating Journal Quality: Is the HIndex a Better Measure Than Impact Factors?".Research on Social Work Practice, vol. 21, $\mathrm{n}^{\circ}$ 2, pp. 222-230. DOI:10.1177/1049731510369141

JACSÓ, P. (2009). "Five-year impact factor data in the Journal Citation Reports". Online Information Review, vol. 33, $\mathrm{n}^{\mathrm{o}}$ 3, pp. 603-614. DOI:10.1108/14684520910969989

JAMALI, J.; SALEHI-MARZIJARANI, M.; AYATOLLAHI, S. (2014).“Factors Affecting Journal Quality Indicator in Scopus (SCImago Journal Rank) in 
Obstetrics and Gynecology Journals: a Longitudinal Study (1999-2013)". Acta Informatica Medica, vol. 22, ${ }^{\circ}$ 6, pp. 385. DOI:10.5455/aim.2014.22.385-388 KIM, J.; HUH, S.; CHU, M.S. (2014). "Correlation among the citation indices of Korean scientific journals listed in international databases". Science Editing, vol. $1, \mathrm{n}^{\mathrm{o}} 1$, pp. 27-36. DOI:10.6087/kcse.2014.1.27

LEYDESDORFF, L. (2009). "How are new citation-based journal indicators adding to the bibliometric toolbox?".Journal of the American Society for Information Science and Technology, vol. 60, $\mathrm{n}^{\mathrm{o}}$ 7, pp. 1327-1336. DOI:10.1002/asi.21024

LÓPEZ-ILLESCAS, C.; MOYA-ANEGÓN, F.; MOED, H. F. (2009). "Comparing bibliometric country-by-country rankings derived from the Web of Science and Scopus: the effect of poorly cited journal in oncology". Journal of Information Science, vol. 35, 244-256

MEHO, L.I.; ROGERS, Y. (2008).“Citation counting, citation ranking, and hindex of human-computer interaction researchers: a comparison of Scopus and Web of Science". Journal of the American Society for Information Science and Technology, vol. 59, no 11, pp. 1711-1726. Doi: 10.1002/asi.20874

MOED, H.F. (2010). "Measuring contextual citation impact of scientific journals".Journal of Informetrics, vol. 4, $\mathrm{n}^{\mathrm{o}}$ 3, pp. 265-277. DOI: 10.1016/j.joi.2010.01.002

OOSTHUIZEN, J.C.; FENTON, J.E. (2013).“Alternatives to the impact factor".Surgeon, vol. 12, no 5, pp. 239-243. DOI:10.1016/j.surge.2013.08.002

RIZKALLAH, J.;SIN, D.D. (2010). "Integrative approach to quality assessment of medical journals using impact factor, Eigenfactor, and Article influence scores".PLOS ONE, vol. 5, $\mathrm{n}^{\mathrm{o}} \quad 4, \quad$ pp. e10204. DOI:10.1371/journal.pone.0010204

ROCHA-E-SILVA, M. (2010)."Impact factor, Scimago Indexes and the Brazilian journal rating system: where do we go from here?".Clinics (Sao Paulo, Brazil), vol. $65, \mathrm{n}^{\circ}$ 4, pp. 351-355. DOI:10.1590/S1807-59322010000400001

ROUSSEAU, R. (2009)." On the relation between the WoS impact factor, the Eigenfactor, the SCImago journal rank, the article influence score and the journal h-index". Conference Proceedings, Nanjing University, pp. 1-13. $<$ http://eprints.rclis.org/13304 $\geq$ [consulta28/06/2015]

SELLERS, S.L.; MATHIESEN, S.G.; PERRY, R.; SMITH, T. (2004)."Evaluation of social work journal quality: Citation versus reputation approaches". Journal of Social Work Education, vol. 40, nº 1, pp. 143-160.

TORRES-SALINAS, D.; JIMÉNEZ-CONTRERAS, E. (2010).“Introducción y estudio comparativo de los nuevos indicadores de citación sobre revistas científicas en JournalCitationReports y Scopus". El Profesional de la Información, vol. 19, $\mathrm{n}^{\mathrm{o}}$ 2, pp. 201-208. DOI:10.3145/epi.2010.mar.12 
UNIVERSITY OF WASHINGTON.(2012). Eigenfactor.org ${ }^{\circledR}$. Ranking and mapping scientific knowledge. <www.eigenfactor.org/methods.php $\geq$ [consulta 20/06/2015]

VANCLAY, J.K. (2008). "Ranking forestry journals using the h-index".Journal of Informetrics, vol. 2, $\mathrm{n}^{\mathrm{o}}$ 4, pp. 326-334. DOI: 10.1016/j.joi.2008.07.002

YIN, C.Y. (2011)."Do impact factor, h-index and Eigenfactor of chemical engineering journals correlate well with each other and indicate the journals' influence and prestige?".CurrentScience, vol. 100, $\mathrm{n}^{\circ}$ 5, pp. 648-653. 